\section{Saturn's Spokes: Lost and Found}

\author{
C. J. Mitchell, ${ }^{1}$ M. Horányi, ${ }^{1 *}$ O. Havnes, ${ }^{2}$ C. C. Porco $^{3}$
}

The spokes are intermittently appearing radial markings in Saturn's B ring that are believed to form when micrometer-sized dust particles are levitated above the ring by electrostatic forces. First observed by the Voyagers, the spokes disappeared from October 1998 until September 2005, when the Cassini spacecraft saw them reappear. The trajectories of the charged dust particles comprising the spokes depend critically on the background plasma density above the rings, which is a function of the solar elevation angle. Because the rings are more open to the Sun now than when Voyager flew by, the charging environment above the rings has prevented the formation of spokes until very recently. We show that this notable effect is capable of stopping spoke formation entirely and restricting the size of the particles in the spokes.

$\mathrm{V}$ oyagers and Cassini have collected images of spokes at Saturn (Fig. 1). These features, which are composed of micrometer-sized dust particles, are typically $10,000 \mathrm{~km}$ in length and $2000 \mathrm{~km}$ in width. Although they last for hours, there are indications that spokes form very rapidly $(1,2)$. Observations of Saturn's rings by the Hubble Space Telescope (HST) (3) started around the time when Earth crossed Saturn's ring plane in 1995 and continued through 2004. The spokes were observed to fade as the ring opening angle $\left(B^{\prime}\right)$ increased, which resulted from Saturn's orbital motion and its tilted axis of rotation changing the light-scattering geometry. The changing geometry accounted for the diminishing visibility of the spokes in the HST observations, because light must pass through the spokes at a shallow angle for them to be observed. On the basis of these arguments, Cassini was expected to see spokes during periods when it was near the ring plane (3). Contrary to these expectations (4), Cassini has not yet seen spokes on the illuminated side of the rings, despite many dedicated observations during its first 15 months in orbit, indicating that the spokes were largely absent. Here, we extend an earlier suggestion that the value of $B^{\prime}$ may affect spoke activity (5) and show that for large values of $B^{\prime}$, spokes cannot form at all.

Electrostatic interactions were suggested early on to explain spoke formation (6-8). The interactions involved a transient event to charge grains to sufficiently large potentials for lift-off from the ring. Meteorite impacts $(6,7)$ or highenergy auroral electron beams $(8)$ could create a region of sufficiently dense plasma immediately above the ring. This short-lived transient plasma charges the boulders of the main ring to a negative potential due to electron collection. A small fraction of the dust grains

${ }^{1}$ Laboratory for Atmospheric and Space Physics, and Department of Physics, University of Colorado, Boulder, CO 80304-0392, USA. ${ }^{2}$ Department of Physics, The Auroral Observatory, University of Tromsø, 9000 Tromsø, Norway. ${ }^{3}$ Cassini Imaging Central Laboratory for $\mathrm{Op}$ erations, Space Science Institute, Boulder, CO 80301, USA.

${ }^{*}$ To whom correspondence should be addressed. E-mail: horanyi@colorado.edu on the surface of the boulders collect an extra electron and are repelled from the surface by the electrostatic forces. For a few seconds after lift-off, the dust grains remain immersed in the short-lived dense plasma and are accelerated away from the ring. Subsequently, they leave the dense transient plasma cloud and enter the permanently present background plasma environment.

Voyager took spoke images that were separated by several minutes and observed fully formed spokes that were absent in the image taken just moments before (2). These observations indicate that spokes could form instantaneously over a large radial distance, as expected if they were triggered by a sheet of high-energy auroral electrons connecting the ionosphere to the ring (8). Alternatively, if spoke formation were triggered by meteorite impacts, the transient plasma cloud would have to drift along the full radial extent of the spokes in minutes $(6,7,9,10)$. Cassini is capable of taking images at a much higher rate than Voyager and will be able to distinguish between these two models.

For our simulations, we assume that the meteorite impact model is correct and use the expected properties of the ambient and transient plasmas to calculate the potential on the ring, the charging of the dust grains, and the electrostatic forces acting on the grains, as well as to follow their trajectories above the ring. The source of the background plasma is believed to be photosputtering off the main ring followed by photoionization of the subsequent neutral exosphere (11). We take the plasma density to be proportional to $\sin \left(B^{\prime}\right)$ and use this density at the maximum opening angle $\left(B_{\text {max }}^{\prime} \cong 27^{\circ}\right)$ as a parameter, $n_{\max }=n_{\mathrm{H}^{+}}\left(B^{\prime}=B_{\max }^{\prime}\right)$, where $n_{\mathrm{H}^{+}}$ is the density of hydrogen ions. During Cassini's orbit insertion in July $2004\left(B^{\prime}=24.4^{\circ}\right)$, the density and the composition of the plasma above the main rings were measured (12). The plasma is composed of $\mathrm{H}^{+}$and $\mathrm{O}_{2}^{+}$, as well as $\mathrm{O}^{+}$ions, with a density of $0.1<n_{\mathrm{H}^{+}}<1 \mathrm{~cm}^{-3}$ and mixing ratios of $n_{\mathrm{H}^{+}}: n_{\mathrm{O}^{+}}: n_{\mathrm{O}^{+}}=1: 0.3: 1$.

The equilibrium potential of the ring itself can be calculated by setting the sum of the currents equal to zero, $\sum_{j} I_{j}=0$, where $I_{j}$ represents the collection of electrons and ions, as well as the production of photoelectrons, all of which are functions of the potential of the ring, $\phi_{\mathrm{r}}$. The ring potential itself is a function of $n_{\max }$, and it remains independent of $B^{\prime}$, because all of the charging currents are proportional to $\sin \left(B^{\prime}\right)$. Based on Cassini plasma measurements, the ring potential is expected to be in the range of $-1.7<\phi_{\mathrm{r}}<1.6 \mathrm{~V}$.

Because of the charging of the ring and the resulting electric field, the electron and ion densities immediately above the ring will not be equal. These densities can be calculated by considering the conservation of energy and flux, resulting in densities for all the species considered (5) as functions of $\phi$. The potential in the plasma as a function of height above the ring is the solution of Poisson's equation

$$
\frac{d^{2} \phi}{d z^{2}}=-4 \pi e\left(n_{\mathrm{H}^{+}}+n_{\mathrm{O}^{+}}+n_{\mathrm{O}_{2}^{+}}-n_{\mathrm{e}}-n_{\mathrm{v}}\right)
$$

where $e$ is the charge of an electron, and where in addition to the ion densities, we also consider

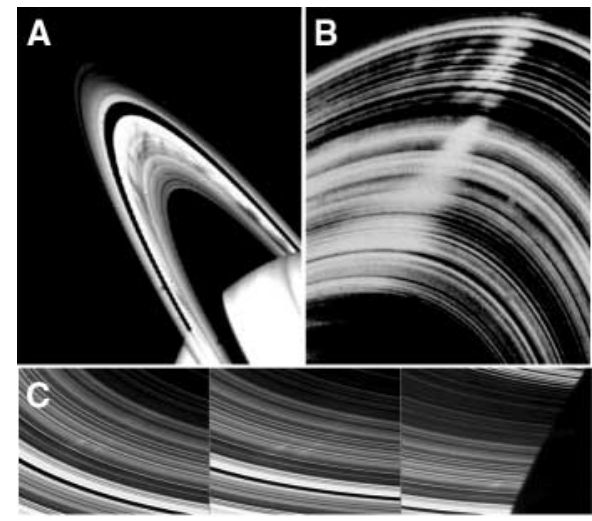

Fig. 1. (A and B) Voyager 2 images of spokes in the $B$ ring $(1,2)$. (A) An image captured in backscattered light before the closest encounter; the spokes appear as dark radial features across the ring plane. (B) An image taken in forward-scattered light after Voyager crossed the ring plane, looking back toward the Sun; the spokes appear as bright markings. Typical dimensions of these spokes are $10,000 \mathrm{~km}$ in length and $2000 \mathrm{~km}$ in width. The changing brightness indicates that spokes consist of small grains with radii comparable to the wavelength of the visible light $(<1 \mu \mathrm{m})$. At the time these images were taken, the rings' opening angle to the sun was $B^{\prime}=8^{\circ}$. (C) The first set of spoke observations by Cassini taken on 5 September $2005\left(B^{\prime}=20.4^{\circ}\right)$ over a span of $27 \mathrm{~min}$. These faint and narrow spokes were seen from the unilluminated side of the $B$ ring. These spokes are $\approx 3500 \mathrm{~km}$ long and $\approx 100 \mathrm{~km}$ wide, much smaller than the average spokes seen by Voyager. These images were taken with a resolution of $17 \mathrm{~km}$ per pixel at a phase angle of $145^{\circ}$ when Cassini was $13.5^{\circ}$ above the dark side of the rings as the spokes were about to enter Saturn's shadow. [Images courtesy NASA/Jet Propulsion Laboratory (JPL)California Institute of Technology and NASA/JPL Space Science Institute] 
the densities of the ambient electrons $\left(n_{\mathrm{e}}\right)$ and the photoelectrons $\left(n_{v}\right)$ released from the ring. The boundary conditions for $\phi$ are $\phi(z=o)=$ $\phi_{\mathrm{r}}$, where $z$ is the height above the ring, and there is no electric field far from the ring so that $\nabla \phi=0$ at $z=10 \lambda_{\mathrm{D}}$, where $\lambda_{\mathrm{D}}$ is the Debye length in the plasma. This length is typically tens of meters at the maximum plasma density and is a function of the plasma density $\left(\lambda_{\mathrm{D}} \sim \sqrt{k T_{\mathrm{e}} / n_{\mathrm{e}}}\right.$, where $k$ is the Boltzmann constant, and $T_{\mathrm{e}}$ is the electron temperature), which varies as $\sin \left(B^{\prime}\right)$. Hence, the electric field, $E \cong \phi_{\mathrm{r}} / \lambda_{\mathrm{D}}$, increases as $B^{\prime}$ increases, even though the potential of the ring, $\phi_{\mathrm{r}}$, does not depend on this angle.

The motion of a spoke particle in one dimension can be followed by simultaneously integrating its equation of motion and the charging currents, including the effects of the electric field in the plasma sheath, gravity from the ring, and the $z$ component of Saturn's gravitational field

$$
\frac{d^{2} z}{d t^{2}}=\frac{-G M z}{r^{3}}-2 \pi G \sigma+\frac{q(t)}{m} E
$$

where $G$ is the gravitational constant, $M$ is Saturn's mass $\left(5.8 \times 10^{29} \mathrm{~g}\right), m$ is the grain's mass, $r$ is the distance from Saturn, $\sigma$ is the ring's surface-mass density $\left(100 \mathrm{~g} / \mathrm{cm}^{2}\right)(13), q$ is the charge on the dust grain, and $E$ is the electric field. Initially, the electric field and the charging currents are set by the transient plasma cloud, and later by the ambient plasma environment.

Because we expect the transient plasma to be short lived, we subjected the dust grains to a dense plasma for a few seconds before switching to the ambient plasma environment. The transient plasma has a density of $n_{\mathrm{T}}=100 \mathrm{~cm}^{-3}$ and is assumed to be dominated by oxygen ions with a temperature of $2 \mathrm{eV}$ (7). Independently of $B^{\prime}$, this produces a negatively charged ring with a potential of about $\phi_{\mathrm{r}} \cong-9 \mathrm{~V}$ and a plasma sheath with a Debye length of $\lambda_{\mathrm{D}} \cong 1 \mathrm{~m}$. Even in this dense plasma, the surface charge density on the ring remains low and most dust particles on the boulders remain uncharged. The small fraction of the grains that collect an electron will be lifted off the ring by the electric field. Although the source of the dense plasma and its dynamics are still being debated $(9,10)$, the details are unimportant to our model because the dust grains will be singly charged and exposed to a short-lived electric field regardless of which formation theory is correct.

For a low ambient plasma density (12), the ring remains at a positive potential, independent of $B^{\prime}$. For example, when $n_{\max }=0.11 \mathrm{~cm}^{-3}, \phi_{\mathrm{r}}=$ $1.5 \mathrm{~V}$, and there is an abrupt change in the lifetime of the dust grains as a function of $B^{\prime}$ (Fig. 2). In the transient plasma, the characteristic time for a grain with a radius of $0.5 \mu \mathrm{m}$ to lose its excess electron and collect an ion is about $10 \mathrm{~s}$. Because this transient plasma is expected to dissipate in seconds (6), most of the small grains will emerge either with their excess negative charge or neutral. Grains that are too large quickly collect an excess ion and immediately fall back down to the ring, never leaving the transient plasma cloud. The subsequent charging of the small grains that escape the transient plasma cloud depends on the ambient plasma density. If this plasma density were high, the grain charging would be dominated by electron collection and the grains would collect a negative charge and fall back down to the ring, which by this time would have returned to its usual positive potential. In a low plasma density environment, the small grains would charge positively, due to the dominating photoelectron current. In this case, the grains continue to be repelled by the ring and can form spokes.
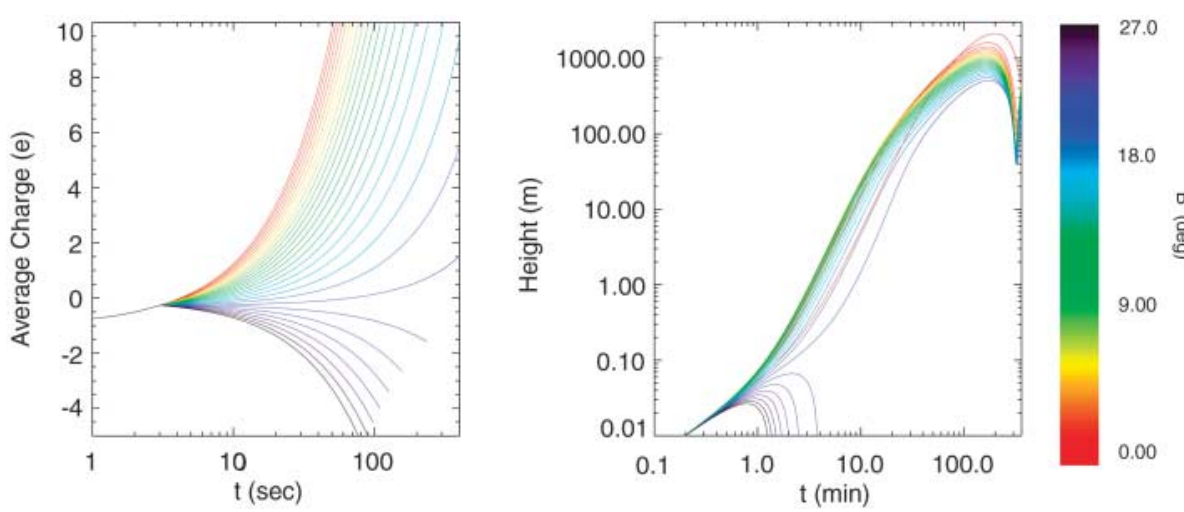

We followed the trajectories of grains with radii up to $10 \mu \mathrm{m}$ in diameter (Fig. 3). For $n_{\max }=$ $0.11 \mathrm{~cm}^{-3}$, there is an abrupt increase of the lifetime as $B^{\prime}$ drops below $20^{\circ}$, indicating the transition between periods when spokes cannot form and when they will be abundant. The value of $B^{\prime}$ at which the spokes turn on and off is a function of $n_{\max }$. From the Cassini plasma measurements (12), the maximum plasma density is in the range of $0.1<n_{\max }<1 \mathrm{~cm}^{-3}$. Taking $n_{\max }=0.11 \mathrm{~cm}^{-3}$, we can match the Cassini imaging observations of the recent reappearance of the spokes in September of 2005 on the unilluminated rings if we assume that these dark-side spoke sightings are indeed observations of day-side spokes seen through the rings. For different values of $n_{\max }$, the behavior can be markedly different. For example, for $n_{\text {max }} \approx 0.23 \mathrm{~cm}^{-3}, \phi_{\mathrm{r}} \cong 0$, and spokes would form for any value of $B^{\prime}$. For even larger values of $n_{\max }$, spokes would form only for large values of $B^{\prime}$, contradicting the observations. For the range $0.1<n_{\max }<0.2$, the onset of the spoke activity shifts by several degrees and the higher plasma densities reduce the range of $B^{\prime}$ where spokes can form.

The upper cut-off in the lifetime of particles as a function of their size depends on the properties of the transient plasma. The larger particles that lose their excess electron too quickly are rapidly pulled back down to the ring without ever leaving the transient plasma cloud. This is consistent with the photometry of the spokes indicating the lack of particles bigger than a few micrometers (14).

The sudden reappearance of the spokes to Cassini at $B^{\prime} \cong 20^{\circ}$ indicates that the plasma density above the rings must be close to the lower limit indicated by the in situ measurements (12). Notably, if spoke activity switches

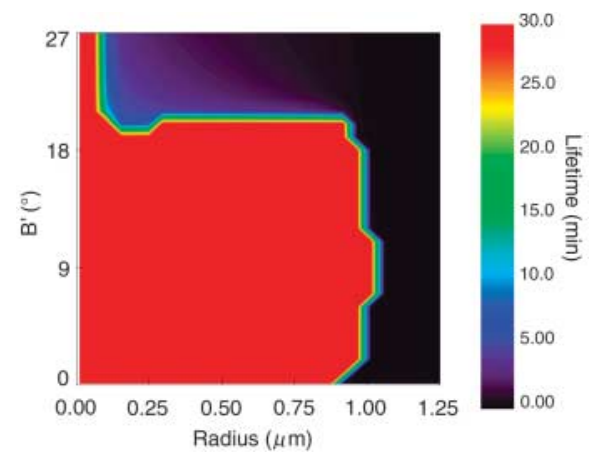

Fig. 3. The lifetime of dust particles above the ring as a function of $B^{\prime}$ and particle size. The abrupt change in the lifetime of the particles with radii $0.1<a<1 \mu \mathrm{m}$, as indicated by the sharp color transition from red to purple at $B^{\prime}=20^{\circ}$, indicates that spoke activity is expected to turn on and off rapidly as a function of $B^{\prime}$. The exact value at which this transition happens is a function of the ambient plasma density $n_{\max }$. The cut-off for large particles is a function of the amount of time the grains spend in the transient plasma. 
off for $\left|B^{\prime}\right|>20^{\circ}$, we expect spoke activity for about 8 years at a time, followed by a period without spokes that lasts 6 to 7 years. Within a few days of the dark-side spoke sightings on 5 September (Fig. 1), Cassini captured several series of excellent images of the lit side without any indications of spokes. Hence, the recently seen spokes might just represent an "early-bird" event, before the onset of the main spoke season. If these 5 September spokes were indeed the start of the main season, the background plasma density must be on the order of $n_{\max } \approx$ $0.1 \mathrm{~cm}^{-3}$. However, if the HST observations of the last spoke sighting $\left(B^{\prime}=15^{\circ}\right)$ correspond to the start of the off season, this would require $n_{\text {max }} \approx 0.2 \mathrm{~cm}^{-3}$, and spoke activity will not be expected to return until late 2006. In summary, spokes are expected to be seen if the plasma conditions are favorable for their formation and either the observer or the Sun is near the ring plane. Currently, Cassini is orbiting too close to the ring plane and cannot make any observations. We expect that spoke activity will have returned by the time its inclination increases again in July of 2006.

\section{References and Notes}

1. B. A. Smith et al., Science 212, 163 (1981).

2. B. A. Smith et al., Science 215, 504 (1982).

3. C. A. McGhee et al., Icarus 173, 508 (2005).

4. M. Horányi, T. W. Hartquist, O. Havnes, D. A. Mendis, G. E. Morfill, Rev. Geophys. 42, RG4002 (2004).

5. T. Nitter, O. Havnes, F. Melandsø, J. Geophys. Res. 103, 6605 (1998).
6. G. E. Morfill, C. K. Goertz, Icarus 55, 111 (1983).

7. C. K. Goertz, G. Morfill, Icarus 53, 219 (1983).

8. J. R. Hill, D. A. Mendis, Moon Planets 24, 431 (1981).

9. A. J. Farmer, P. Goldreich, Icarus 179, 535 (2005).

10. G. E. Morfill, H. M. Thomas, Icarus 179, 539 (2005).

11. R. L. Tokar et al., Geophys. Res. Lett. 32, L14S04 (2005).

12. J. H. Waite Jr. et al., Science 307, 1260 (2005).

13. J. N. Cuzzi et al., in Planetary Rings, R. Greenberg, A. Brahic, Eds. (Univ. of Arizona Press, Tucson, AZ, 1984), pp. 73-199.

14. L. R. Doyle, E. Grün, Icarus 85, 168 (1990).

15. This work was supported by the Cassini project. We benefited from discussions on ring dynamics at the Second Midnight Sun Workshop in Tromsø, supported by the Norwegian Research Council.

13 December 2005; accepted 13 February 2006 10.1126/science. 1123783

\section{Visualizing Picometric}

Quantum Ripples of Ultrafast Wave-Packet Interference

Hiroyuki Katsuki, ${ }^{1,2,3}$ Hisashi Chiba, ${ }^{1,2}$ Bertrand Girard, ${ }^{4}$ Christoph Meier, ${ }^{4 *}$ Kenji Ohmori ${ }^{1,2,3 *}$

Interference fringes in vibrating molecules are a signature of quantum mechanics, but are often so short-lived and closely spaced that they elude visualization. We have experimentally visualized dynamical quantum interferences, which appear and disappear in less than 100 femtoseconds in the iodine molecule synchronously with the periodic crossing of two counterpropagating nuclear wave packets. The obtained images have picometer and femtosecond spatiotemporal resolution, representing a detailed picture of the quantum interference.

$\mathrm{I}$ nterference is a clear manifestation of the quantum-mechanical wave character of a physical system $(1,2)$. It has been visualized in the past decade through compelling experiments involving the translational degree of freedom of atoms (3-6), complex molecules (7), and even Bose-Einstein condensates (8).

Interferences have also been observed in the relative motion of the nuclei in molecules. In predissociative states, partial reflection of the oscillating wave packet produces a sequence of equally spaced partial wave packets on the dissociative part of the potential. Interferences of these wave packets are a direct consequence of the natural spreading of matter waves (9). Interferences can also be produced by creating a sequence of two partial wave packets on the same dissociative potential. Again, overlap and interferences result from natural spreading of the wave packets during their propagation $(10,11)$.

${ }^{1}$ Institute for Molecular Science, Myodaiji, Okazaki 444-8585, Japan. ${ }^{2}$ Core Research for Evolution of Science and Technology, Japan Science and Technology Agency, Japan. ${ }^{3}$ The Graduate University for Advanced Studies (SOKENDAI), Myodaiji, Okazaki 444-8585, Japan. 'Laboratoire Collisions, Agrégats, Réactivité, CNRS UMR 5589, Institut de Recherche sur les Systèmes Atomiques et Moléculaires Complexes, Université Paul Sabatier-Toulouse 3, France.

*To whom correspondence should be addressed. E-mail: chris@irsamc.ups-tlse.fr (C.M.), ohmori@ims.ac.jp (K.O.)
In all these examples, the partial wave packets are propagating in the same direction, and the interference pattern, which involves components from the different partial waves having the same momentum, is very stable in time. The situation for counterpropagating wave packets is completely different. The interference pattern is localized in space only during the crossing time. High temporal and spatial resolutions, and strong robustness toward averaging effects, are required to observe it. A wave packet oscillating in an anharmonic well provides a natural situation for such interferences.

When several quantum states of a weakly anharmonic system are excited coherently, the time evolution of such a system is characterized by a "revival" phenomenon: An initially well-localized wave packet at first disperses, and then relocalizes after some well-defined revival time and regains its original shape. This effect had been theoretically predicted (12-14) and was later experimentally verified for a large number of very different quantum systems, e.g., atomic Rydberg states $(15,16)$ or mobroadband ultrashort laser pulses. Furthermore, at a certain, well-defined earlier time (halfrevival time), the wave packet consists of two copies of the initial wave packet, shifted by half a vibrational period. As has been shown theoretically (25), in this special situation, the lecular vibrational levels (17-24) excited by two counterpropagating wave packets produce pronounced interference structures when they cross. Additionally, a detailed theoretical treatment shows that the fringes are out of phase for subsequent crossing events.

Here, we use a laser pump-probe scheme to visualize this event. The interaction with the pump pulse centered around $587 \mathrm{~nm}$ creates a vibrational wave packet in the electronic B state of $\mathrm{I}_{2}$, widely studied in the gas phase (17-21) as

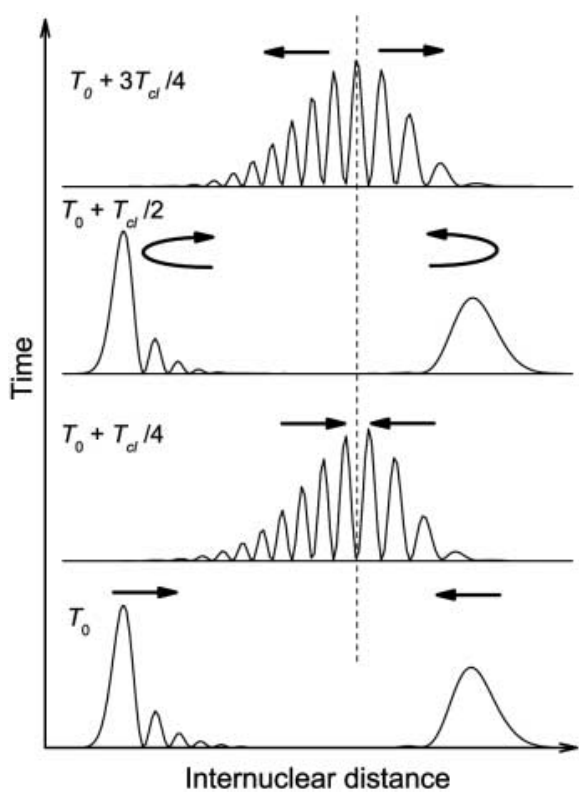

Fig. 1. Evolution of the two wave packets (probability distributions $|\Psi(r, T)|^{2}$ ) around the halfrevival time $\left(T_{0}=p T_{\mathrm{cl}} \cong T_{\text {rev }} / 4, p\right.$ integer $)$. At $T_{0}$, the two wave packets are localized at the turning points and start to propagate toward each other. At $T_{0}+T_{\mathrm{cl}} / 4$, the two wave packets overlap and interfere, creating a stationary wave that lasts during their whole crossing. At $T_{0}+T_{\mathrm{cl}} / 2$, the two wave packets are at the opposite turning points and start to come back. At $T_{0}+3 T_{\mathrm{cl}} / 4$, the wave packets intersect once more. The stationary wave resulting from their interference is phase shifted by $\pi$ as compared to the previous crossing (see the vertical dashed line). 